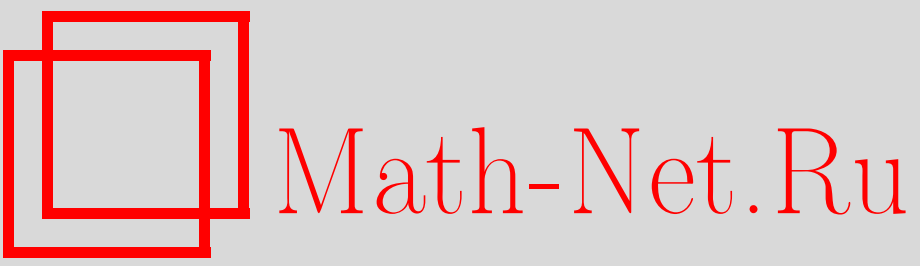

Н. Н. Андреев, Один сферический код, УМН, 1999, том 54, выпуск 1, 255-256

DOI: https://doi.org/10.4213/rm123

Использование Общероссийского математического портала Math-Net.Ru подразумевает, что вы прочитали и согласны с пользовательским соглашением

http://www.mathnet.ru/rus/agreement

Параметры загрузки:

IP : 54.198 .67 .100

26 апреля 2023 г., 10:10:15 


\title{
ОДИН СФЕРИЧЕСКИЙ КОД
}

\author{
Н.Н. АнДРЕЕВ
}

Пусть $S^{d-1}=\left\{\left(x_{1}, x_{2}, \ldots, x_{d}\right) \in \mathbb{R}^{d} \mid x_{1}^{2}+x_{2}^{2}+\cdots+x_{d}^{2}=1\right\}$ - единичная сфера в $d$-мерном евклидовом пространстве; для $x, y \in \mathbb{R}^{d}$ через $x y$ обозначим скалярное произведение векторов.

Конечное подмножество $W$ сфферы $S^{d-1} \subset \mathbb{R}^{d}$ называется $\tau$-кодом, если $x y \leqslant \tau$ для всех $x, y \in W, x \neq y$. Представляет болшшой интерес вопрос отыскания при заданных $d$ и $\tau$ кодов, содержащих наибольшее количество точек. При $\tau=1 / 2$ это хорошо известная задача о контактном числе шаров, решение которой известно только при $d=2,3,8,24$ [ 1 ; с. 45]. В этой заметке будет рассматриваться $d=4, \tau=\cos (\pi / 5)$.

Через $W_{120}$ будем обозначать множество вершин правильного многогранника в $\mathbb{R}^{4} \mathrm{c}$ символом Шлефли $\{3,3,5\}$ [2; с. 494]. Его 120 вершин состоят из: 8 вершин вида $( \pm 1,0,0,0),(0, \pm 1,0,0),(0,0, \pm 1,0),(0,0,0, \pm 1) ; 16$ вершин вида $( \pm 1 / 2, \pm 1 / 2, \pm 1 / 2, \pm 1 / 2)$; и 96 вершин, полученных из 8 точек вида $( \pm(\sqrt{5}+1) / 4, \pm 1 / 2, \pm(\sqrt{5}-1) / 4,0)$ четными перестановками координат. Множество скалярных произведений $x y, x, y \in W_{120}$, есть $\{-1, \cos (4 \pi / 5), \cos (2 \pi / 3), \cos (3 \pi / 5), 0, \cos (2 \pi / 5), \cos (\pi / 3), \cos (\pi / 5), 1\}$. Таким образом, $W_{120}$ является $\cos (\pi / 5)$-кодом. Покажем, что он содержит максимальное количество точек, возможное для множества, являющегося таким кодом.

Tеорема. Пусть $W=\left\{x^{(i)}\right\}_{i=1}^{N} \subset S^{3}-\cos (\pi / 5)$-код с максимально возможсным числом точек. Тогда $N=120$.

ДокаЗАТЕльство. Оценку снизу дает приведенная конструкция $W_{120}$.

Для оценки сверху рассмотрим систему многочленов Гегенбауэра $\left\{P_{\nu}(t)\right\}_{\nu=0}^{\infty}$, ортогональную на интервале $(-1,1)$, с весом $\left(1-t^{2}\right)^{1 / 2}$ и с нормировкой $P_{\nu}(1)=1$ :

$$
P_{0}(t)=1, P_{1}(t)=t, \ldots ; \quad(\nu+2) P_{\nu+1}(t)=(2 \nu+2) t P_{\nu}(t)-\nu P_{\nu-1}(t) .
$$

В геометрических задачах, начиная с работы [3] $\Phi$. Дельсарта, используется положительная определенность многочленов Гегенбауэра [1; с. 318]: для любого конечного множества точек $x^{(1)}, \ldots, x^{(N)}$ из $S^{3}$, любого $\nu \in \mathbb{N}$ и любых $p_{k}, p_{l} \in \mathbb{C}$ справедливо неравенство $\sum_{k, l=1}^{N} P_{\nu}\left(x^{(k)} x^{(l)}\right) p_{k} \overline{p_{l}} \geqslant 0$. Получение оценки сверху будет проводиться по схеме Дельсарта, развивавшейся в работах [4]-[10], где она применялась для оценок плотности упаковок, контактных чисел и в некоторых других задачах, связанных с экстремальным расположением точек на сфере.

$$
\begin{aligned}
& \text { Для доказательства теоремы потребуется полином 17-й степени } \\
& \begin{aligned}
h(t)=t^{2}(t+1)^{2}\left(t^{2}-1 / 4\right)^{2}\left(t^{2}-(3-\sqrt{5}) / 8\right)^{2}(t+(1+\sqrt{5}) / 4)^{2}(t-(1+\sqrt{5}) / 4) \\
\quad \times\left(65536(232 \sqrt{5}-303) t^{2}+8192(3079-2990 \sqrt{5}) t+2048(4255 \sqrt{5}-1777)\right) .
\end{aligned}
\end{aligned}
$$

В его разложении по многочленам Гегенбауэра $h(t)=\widehat{h}_{0} P_{0}(t)+\widehat{h}_{1} P_{1}(t)+\cdots+\widehat{h}_{17} P_{17}(t)$, все $\widehat{h}_{\nu}$ неотрицательны и для полноты рассуждений приведены в конце доказательства.

В дальнейшем будут использоваться следующие свойства этого полинома:

1) $\widehat{h}_{0}>0, \widehat{h}_{\nu} \geqslant 0$ для $\nu=1,2, \ldots, 17$

2) $h(t) \leqslant 0$ при $t \in[-1, \cos (\pi / 5)]$ (следует из вида полинома, так как $\cos (\pi / 5)=(1+\sqrt{5}) / 4$, а последний множитель не имеет действительных корней и положителен);

3) $h(1) / \widehat{h}_{0}=120$.

Так как конструкция полинома существенно опирается на схему доказательства, приведем схему Дельсарта в том виде, который потребуется для дальнейшего. Пусть на $S^{3}$ дан $\cos (\pi / 5)$-код $W=\left\{x^{(i)}\right\}_{i=1}^{N}$, состоящий из $N$ точек. Используя первое отмеченное свойство полинома $h(t)$ и положительную определенность многочленов Гегенбауэра, имеем:

$$
\sum_{k, l=1}^{N} h\left(x^{(k)} x^{(l)}\right)=\sum_{\nu=0}^{\infty} \widehat{h}_{\nu} \sum_{k, l=1}^{N} P_{\nu}\left(x^{(k)} x^{(l)}\right) \geqslant \widehat{h}_{0} \sum_{k, l=1}^{N} 1=N^{2} \widehat{h}_{0} .
$$

Работа выполнена при финансовой поддержке РФФИ-ГФЕН (грант № 96-01-00036С). 
Так как многочлен $h(t)$ неположителен на отрезке $[-1, \cos (\pi / 5)]$, а $x^{(k)} x^{(l)} \leqslant \cos (\pi / 5)$ для $x^{(k)}, x^{(l)} \in W$, то эту же сумму можно оценить с другой стороны:

$$
\sum_{k, l=1}^{N} h\left(x^{(k)} x^{(l)}\right)=\sum_{k=1}^{N} h\left(x^{(k)} x^{(k)}\right)+\sum_{k, l=1, k \neq l}^{N} h\left(x^{(k)} x^{(l)}\right) \leqslant N h(1) .
$$

Таким образом, $N \leqslant h(1) / \widehat{h}_{0}$. Подставляя значения $h(1)=505800$ и $\widehat{h}_{0}=4215$, получаем требуемую оценку $N \leqslant 120$.

Значения коэффициентов Фурье полинома $h(t)$ по системе многочленов Гегенбауэра:

$$
\begin{aligned}
& \widehat{h}_{0}=4215, \quad \widehat{h}_{1}=5(5941+319 \sqrt{5}) / 2, \quad \widehat{h}_{2}=3(87653+1682 \sqrt{5}) / 8, \\
& \widehat{h}_{3}=49141+2581 \sqrt{5}, \quad \widehat{h}_{4}=5(105709+3886 \sqrt{5}) / 8, \quad \widehat{h}_{5}=74982+2697 \sqrt{5}, \\
& \widehat{h}_{6}=7(43854+551 \sqrt{5}) / 4, \quad \widehat{h}_{7}=68652-928 \sqrt{5}, \quad \widehat{h}_{8}=9(24747-1682 \sqrt{5}) / 4, \\
& \widehat{h}_{9}=5(15737-2233 \sqrt{5}) / 2, \widehat{h}_{10}=11(7887-1682 \sqrt{5}) / 4, \quad \widehat{h}_{11}=165(73-29 \sqrt{5}), \\
& \widehat{h}_{12}=0, \quad \widehat{h}_{13}=0, \quad \widehat{h}_{14}=15(-2267+1682 \sqrt{5}) / 8, \\
& \widehat{h}_{15}=-3388+3132 \sqrt{5}, \quad \widehat{h}_{16}=85(-11+116 \sqrt{5}) / 8, \quad \widehat{h}_{17}=-2727+2088 \sqrt{5} \text {. }
\end{aligned}
$$

ЗАмЕЧАНИЕ 1. Полином $h(t)$ строился по угаданной экстремальной конструкции. Из схемы доказательства видно, что $h(t)$ может иметь отличными от нуля только те коэффициенты $\widehat{h}_{\nu}$, для номеров $\nu$ которых $\sum_{x, y} P_{\nu}(x y)=0$, где сумма берется по $x, y$ из экстремальной конструкции. Построение функций, неположительных на заданном отрезке и имеющих неотрицательные коэффициенты Фурье по системе многочленов Гегенбауэра с заданными номерами, и со всеми остальными коэффициентами равными нулю, является интересной и до конца не решенной задачей.

ЗАмечАние 2. Существует целое семейство экстремальных многочленов 17-го порядка, минимизирующих функционал $h(1) / \widehat{h}_{0}$ и удовлетворяющих свойствам 1,2 . Все они получаются заменой у рассмотренного многочлена $h(t)$ квадратного трехчлена, не имеющего корней на отрезке $[-1,1]$, стоящего последним сомножителем в определении $h(t)$, на множитель $a t^{2}+b t+c$, где $c=(19603456-7790592 \sqrt{5}+(4425-135 \sqrt{5}) a) / 4260, b=(1441792 \sqrt{5}-3571712+(27 \sqrt{5}-$ $1737) a) / 852,65536(232 \sqrt{5}-303) / 4215 \leqslant a \leqslant 16384(1297-273 \sqrt{5}) / 2685$. Такие квадратные трехчлены также не имеют корней на $[-1,1]$, и, следовательно, свойство 2 выполняется, а соответствующий выбор $a, b$ и $c$ обеспечивает выполнение первого и третьего свойств многочлена $h(t)$.

Автор выражает искреннюю благодарность В. А. Юдину, из обсуждений с которым возникла эта заметка, а также А. Г. Бабенко за полезные замечания.

\section{СПИСОК ЛИТЕРАТУРЫ}

[1] Конвей Дж., Слоэн Н. Упаковки шаров, решетки и группы. Т. 1. М.: Мир, 1990. [2] Берже М. Геометрия. Т. 1. М.: Мир, 1984. [3] Delsarte Ph. // Philips Res. Rep. 1972. V. 2. P. 272-289. [4] Delsarte P., Goethals J. M., Seidel J. J. // Geom. Dedicata. 1977. V. 6. P. 363-388. [5] Кабатянский Г. А., Левенштейн В. И. // Проблемы передачи информации. 1978. T. 14. № 1. C. 3-25. [6] Odlyzko A. M., Sloane N. J. A. // J. Combin. Theory. Ser. А. 1979. V. 26. Р. 210-214. [7] Левенштейн В. И. // Докл. АН СССР. 1979. Т. 245. С. 1299-1303. [8] Сидельников В.М.// Проблемы передачи информации. 1980. Т. 16. №3. С. 17-30. [9] Юдин В. А. // Дискр. матем. 1992. Т. 4. № 2. С. 115-121. [10] А рестов В. В., Бабенко А. Г. // Труды МИРАН. 1997. Т. 219. С. 44-73.

Московский государственный университет им. М. В. Ломоносова E-mail: andreev@mech.math.msu.su Home page: http://mech.math.msu.su/ ${ }^{\sim}$ andreev
Принято редколлегией 10.12 .1998 